\title{
SIMPLE METHOD FOR CALCULATING ISLAND WIDTHS
}

John R. Cary, a James D. Hanson, b B. A. Carreras, ${ }^{\mathfrak{c}}$ and V. E. Lynchc

ABSTRACT

A simple method for calculating magnetic island widths has been developed. This method uses only information obtained from integrating along the closed field line at the island center. Thus, this method is computationally less intensive than the usual method of producing surfaces of section of sufficient detail to locate and resolve the island separatrix. This method has been implemented numerically and used to analyze the buss work islands of ATF. In this case the method proves to be accurate to at least within $30 \%$.

\section{Introduction}

In the design of helical magnetic confinement systems, it is desirable to obtain a system with nested flux surfaces. That is, one would like there to be neither chaotic regions nor islands. The Cary-Hanson technique ${ }^{1}$ allows one to reduce systematically the size of islands while preserving other desirable features of the system, such as reasonable rotational transform, shear, and magnetic well. However, practical constraints, such as power supplies and available space for coil locations, may preclude the complete elimination of islands. Thus, one must calculate the island widths for a given design in order to determine whether it is acceptable. Such calculations are also necessary for comparisons of theory with experimentally observed islands to due, e.g., error fields.

Determination of island widths using standard methods is computationally intensive. Standard methods require the generation of puncture plots with sufficient detail to find a field line just inside, but close to the island boundary. Then, this field line must be integrated for a sufficiently long time for the points in the surface of section to sample the island boundary well enough that the width can be measured. We have developed a new, computationally less intensive method for calculating the island width. Our method uses only information obtained from integrating along the closed field line at the center of the island.

The method works as follows. Fixed points of the return map, the map of a poloidal plane onto itself obtained by following a field line, are found. Such fixed points are the intersection of a closed field line and the surface of section. The map is linearized about this fixed point. This linearized map, or tangent map, describes the motion of points near the fixed point. The trace of the matrix representing the tangent map determines the eigenvalues of the linearized motion. Complex, unit-magnitude eigenvalues imply that the fixed point is elliptical, and the argument of these eigenvalues determines the rotation rate about the island center. In the small-island approximation, this rotation rate depends on the island width and the shear of the underlying

Department of Astrophysical, Planctary, and Atmospheric Scicnecs and Department of Physics, University of Colorado, Boulder, Colorado, 80309-0391 USA.

b Department of Physics, Auburn University, Aubum, Alabama, 36849 USA.

c Fusion Encrgy Division, Oak Ridge National Laboratory, Oak Ridge, Tennessee 37831 USA. 
integrable system. The off-diagonal elements of the tangent map, suitably averaged over the iterates of the fixed point, give the shear. Thus, from the tangent map we are able to deduce the island width.

This method has been implemented in the stellarator design code, IFT (IntegrableField Torsatron). This meihod was used to analyze the buss work ${ }^{2}$ islands of ATF ${ }^{3}$ These islands are moderately well separated. This comparison shows that this method of calculation is accurate to approximately $20 \%$ for this case.

\section{Return map and fixed points}

The systems we are considering are assumed to be mo-fold symmetric in the toroidal angle to lowest order. Breaking of this symmetry is due to small effects such as coil errors and the ambient magnetic field. The magnetic field-line map is generated as follows. One begins' at some point $X \equiv(R, Z)$ in a particular poloidal plane defined to be $\varphi=0$. From this point, a field line is followed by numerically integrating the field line equations until $\varphi=2 \pi / \mathrm{m}_{0}$. At this value of $\varphi$, the poloidal position is $F_{0}=$ $\left(F_{0 R}(R, Z), F_{0 Z}(R, Z)\right)$. This defines the map for the first period of the torus. There are mo such maps, one for each of the periods of the torus. If the periodic symmetry is exact, then these maps are identical.

A fixed point of the map is the intersection of a closed field line with the surface $\varphi=0$. To find fixed points we first introduce the composed map,

$$
F^{n}(X) \equiv F_{n-1}\left(F_{n-2}\left(\ldots F_{0}(X) \ldots\right),\right.
$$

in which the convention, $\mathrm{F}_{\mathrm{i}+\mathrm{m}_{0}}=\mathrm{F}_{\mathrm{i}}$, is used. In the mol-symmetric systems, a $k^{\text {th }}$. order fixed point, $X_{0}$, is a solution to $F^{n}\left(X_{0}\right)=X_{0}$ with $n=k$. In the nonsymmetric systems, a $k^{\text {th }}$-order fixed point is a solution to $F^{n}\left(X_{0}\right)=X_{0}$ with $n=k m_{0}$. (Note that symmetry breaking may change the order of a fixed point.) In either case such fixed points may be found by Newton's method. Once the fixed point is found, its iterates, defined by $\mathbf{X}_{\mathrm{i}+1} \equiv \mathbf{F}_{\mathrm{i}}\left(\mathbf{X}_{\mathrm{i}}\right)$ may be obtained.

Motion of points near the fixed point is governed by the tangent map,

$$
\mathrm{T}_{\mathrm{i}} \equiv\left(\begin{array}{l}
\frac{\partial F_{i R}}{\partial R} \frac{\partial F_{i R}}{\partial Z} \\
\frac{\partial F_{i Z}}{\partial R} \frac{\partial F_{i Z}}{\partial Z}
\end{array}\right) .
$$

We define the partial composition of the tangent maps via $T^{0} \equiv \mathbf{I}$, the identity, and $\mathrm{T}^{\mathrm{k}} \equiv \mathrm{T}_{\mathrm{k}} \mathrm{T}^{\mathrm{k}-1}$. Composing the tangent maps, $\mathbf{M}_{0}=\mathbf{T}_{\mathbf{n}-1} \cdot \mathbf{T}_{\mathbf{n}-2} \cdot \ldots \cdot \mathbf{T}_{0}$, for the entire closed field line yields the motion of nearby points around the initial point of the closed field line.

This tangent map has been extensively studied. (See, e.g., Ref. 4.) When islands are small, the residue, $R=\left[2-\operatorname{Tr}\left(\mathbf{M}_{0}\right)\right] / 4$, is small. This provides the basis for elimination of islands in three-dimensional systems. The angular rotation about the elliptic fixed points, those with $0<R<1$, per $n$ mappings is given by

$$
\delta=\arccos (1-2 R) .
$$

The motion of points near the fixed point has a quadratic invariant, $5 \delta \times \cdot W_{0} \bullet \delta X$, where $W_{0 i j}=\frac{1}{2}\left[\left(M_{0^{\circ}} J\right)_{i j}+\left(M_{0} J\right)_{j i}\right]$, and $\mathbf{J}$ is the fundamental symplectic matrix, $\mathbf{J}=$ $\left(\begin{array}{cc}0 & 1 \\ -1 & 0\end{array}\right)$. Diagonalization of $W_{0}$ yields the 
principal axes of the elliptical invariant curves. The corresponding eigenvalues are $w_{0 \perp}$ and $w_{0 l l}$ with $w_{0 \perp}$ being the larger in magnitude. (In the limit of vanishing island the perpendicular direction is perpendicular to the flux surfaces.) We denote the corresponding unit vectors by $\mathrm{e}_{\mathrm{O}} \perp$ and $\mathrm{e}_{\mathrm{O}} \mathrm{ll}$.

\section{Single-resonance model}

Field line flow is a Hamiltonian system. 6 Thus, to understand the perturbation of flux surfaces away from the ideal nested case, it is sufficient to study perturbed Hamiltonian systems. The single-resonance model for perturbation of a Hamiltonian provides an accurate description of the major islands in a perturbed Hamiltonian system when the neighboring islands are far from overlapping the island being considered. In this model, the Hamiltonian is

$\mathrm{H}=\mathrm{H}_{0}(\psi)+\varepsilon(\psi) \cos (\theta-\mathrm{m} \varphi-\zeta(\mathrm{I}))$,

where $\psi$ is the momentum variable, $\theta$ is the angle variable, and $\varphi$ is the time. The first term in this Hamiltonian is the integrable piece, which corresponds to the system with perfect flux surfaces. The second piece induces islands. [In general, the cosine in (2) may be some other periodic function. However, typically the lowest harmonic is dominant, so that only the cosine is needed.] This Hamiltonian (2) has been extensively analyzed. ${ }^{7}$ Given the limitations of space we simply state the results of this analysis.

Because the Hamiltonian depends on the angles only through the combination, $\eta=\theta-m \varphi / h$ phase-space structures, such as the island, must have this same dependence. This implies that such structures move $\left(\theta=z_{r} \varphi+\theta_{0}\right)$ with the velocity, $t_{r} \equiv m / l$. The resonant value of the momentum (or flux) variable, $\psi_{\mathrm{r}}$, is the value at which the unperturbed motion is in resonance with the perturbation, i.e., $t\left(\psi_{r}\right) \equiv \frac{d_{0}}{d l}\left(\psi_{r}\right)=t_{r}$. Without loss of generality it is assumed that $\varepsilon\left(\psi_{r}\right)>0$.

The width of the island for the Hamiltonian (2) is found by calculating the width of the separatrix. The standard calculation yields the full-width, $\Delta \psi=4\left|\varepsilon / t^{\prime}\right| 1 / 2$, the extent of the separatrix at the o-point, in terms of the resonance amplitude and the shear, $t^{\prime}\left(I_{r}\right)$. The frequency of rotation about the o-poirit is found by linearizing the motion near the o-point, and diagonalizing the appropriate matrix. This procedure give a frequency, $\delta=\left[\left|\varepsilon\left(\psi_{\mathrm{r}}\right) \mathrm{t}^{\prime}\left(\psi_{\mathrm{s}}\right)\right|^{1 / 2}\right.$, for oscillation about the o-point. From this relation and its predecessor, the amplitude of the perturbation may be eliminated to give $\left.\Delta \psi=4 \delta / / \mathrm{lt}^{\prime}\left(\psi_{\mathrm{r}}\right)\right)_{\text {. Th }}$ This is the width in the flux variable $\psi$. To obtain the width, $s$, in distance, we use must divide by the gradient of $\psi$. This gives

$$
s=\Delta \psi /|\nabla \psi|=4 \delta /|c| \nabla \mathfrak{t} \mid
$$

Thus, we have the island width in terms of the shear and the rotation $\delta$, which can be calculated from the residue via Eq. (1).

\section{Calculation of the shear}

The basic idea of the shear calculation is that the tangent map has the form,

$$
\begin{aligned}
M_{0}= & |\nabla \psi|^{2} \mathbf{e}_{0 \perp} \mathbf{e}_{0 \perp}+|\nabla t \| \nabla \theta| \mathbf{e}_{0 \| e_{0 \perp}+} \\
& |\nabla \theta|^{2} \mathbf{e}_{0 \|} \mathbf{e}_{0 \|},
\end{aligned}
$$

to lowest order in the perturbation. Thus, by taking the appropriate element of the tangent map and averaging to obtain the angle 
gradients, one finds the shear. The averaging over the surface is replaced by averaging over the iterates of the fixed point, which to lowest order are equally spaced in the flux angle. (This approximation assumes that the order of the fixed point is large.) Here we present only the result,

$$
|\nabla z|=\frac{\mathbf{e}_{0\|\|} \cdot \mathbf{M}_{0} \cdot \mathbf{e}_{0 \perp}}{\mathrm{C}}
$$

$\left[1+\sum_{\mathrm{k}=1}^{\mathrm{n}} \frac{\mathrm{B}_{T 0}}{\mathrm{~B}_{\mathrm{Tk}} \sqrt{\left(\mathrm{T}^{\mathrm{k} \cdot \mathrm{e}_{0} \mathrm{D}} \cdot \mathrm{W}_{\mathrm{k}} \cdot \mathrm{T}^{\mathrm{k} \cdot \mathrm{e}_{0 \perp}}\right.}}\right]$

in which $C$ is the circumference of the polygon defined by the iterates of the map and $\mathrm{B}_{\mathrm{Tk}}$ is the toroidal field at iterate $\mathrm{k}$. Combination of Eq. (2-4) yields the island width in terms of the tangent map.

\section{Comparison with the buss work} islands of ATF

A surface of section for the buss work islands of ATF is shown in the figure below. We have calculated the island widths using two methods. The results of these two methods are the ordered pairs in the figure. The first of the pair was obtained by the direct method of measuring the width of the island across the o-point from the surface of section. The second was obtained via the calculation outlined here.

This comparison shows that the larger, second-order islands are obtained to within $5 \%$ by this method, while the smaller, thirdorder islands are obtained to within $30 \%$. Likely, the calculation of the third-order islands is less accurate because the presence of the large second-order islands is beginning to make the single-resonance approximation invalid. Conversely, the smaller third-order islands do not affect the second-order islands significantly.

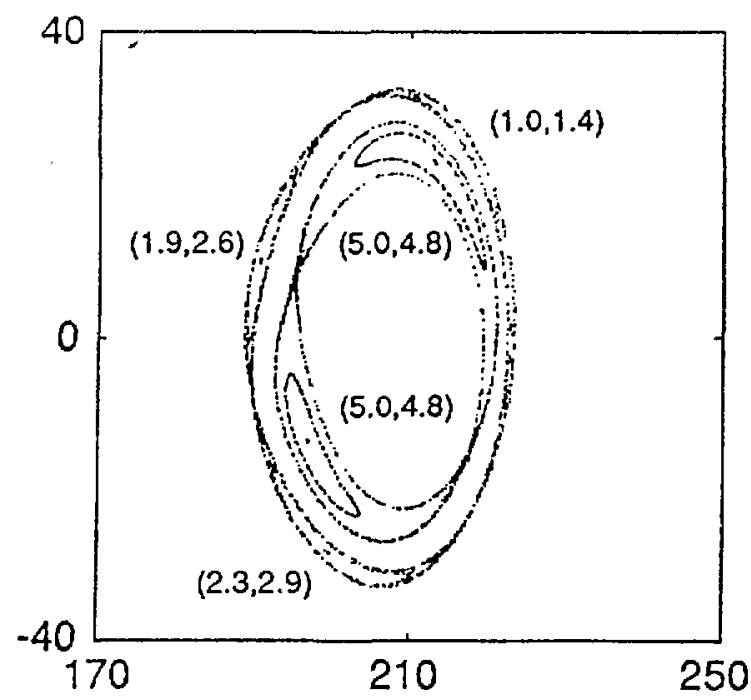

\section{Conclusions}

We have presented a method for calculating island widths using only that information obtained by integrating along the closed magnetic field line at the center of the island. Thus, our method is much less calculationally intensive than the standard method. Our calculation predicts the island widths produced by the buss work of ATF to at least within $30 \%$ accuracy even though these islands are within a factor of two of overlapping.

\section{References}

1 J. D. Hanson and J. R. Cary, Phys. Fluids 27, 767 (1984); J. R. Cary and J. D. Hanson, Phys. Fluids 29, 2464 (1986).

2 D. K. Lec et al, Bull. Am Phys. Soc. 33, 2069 (1988).

3 J.F. Lyon et al, Fusion Technol. 10, 179 (1986).

4 J. M. Grecne, J. Math. Phys. 20, 1183 (1979).

5 A. J. Lichtenberg and M. A. Licberman, Regular and Stochastic Motion (Springer-Verlag, Ncw York, 1983).

6 J. R. Cary and R. G. Littlcjohn, Ann. Phys. (NY) 151, 1 (1983).

7 B. V. Chirikov, Phys. Rep. 52, 263 (1979). 


\section{DISCLAIMER}

This report was prepared as an account of work sponsored by an agency of the United States Government. Neither the United States Government nor any agency thereof, nor any of their employees, makes any warranty, express or implied, or assumes any legal liability or responsibility for the accuracy, completeness, or usefulness of any information, apparatus, product, or process disclosed, or represents that its use would not infringe privately owned rights. Reference herein to any specific commercial product, process, or service by trade name, trademark, manufacturer, or otherwise does not necessarily constitute or imply its endorsement, recommendation, or favoring by the United States Government or any agency thereof. The views and opinions of authors expressed herein do not necessarily state or reflect those of the United States Government or any agency thereof. 